\title{
Method for Group Decision-Making of Multi-Stakeholders with Category Preference on Alternatives
}

\author{
Dai Quan-chen ${ }^{1}$, Zhu Jian-jun ${ }^{1}$ and Zhang Shi-tao ${ }^{1,2}$ \\ ${ }^{1}$ College of Economics and Management, Nanjing University of Aeronautics and \\ Astronautics, Nanjing 211106, China;2. School of Mathematics \& Physics Science \\ and Engineering, Anhui University of Technology, Ma'anshan, 243002,China \\ ${ }^{1}$ daiquanchen@nuaa.edu.cn, ${ }^{2}$ zhujianjun@nuaa.edu.cn; ${ }^{3} 117524212 @ q q . c o m$
}

\begin{abstract}
This paper studies the issue of decision making on some alternatives with category preference of multiple decision makers in which interest conflicts exist and the weights are unknown. It puts forward the method to firstly calculate the attribute weight in each decision maker's circumstance through case-based learning and thus meet subjective preferences of different decision makers. Considering the interest conflicts between multiple decision makers, the fuzzy set theory is then used to optimize the weight of each decision makers to comply with objective requirements of group decision-making process. Finally, the total order relation of the alternative which does not only conform to the subjective preference information of the decision maker but also consist with the objective fact of group decision-making is obtained based on the calculation result of overall offtarget distance for the alternative. The case indicates application procedures and method feasibility.
\end{abstract}

Keywords: Multiple decision makers; alternatives with category preference; multiattribute; group decision-making; grey target decision making

\section{Introduction}

With the development of modern society, decision-making problems in social and economic activities have become more and more complicated and volatile. It is increasingly difficult to make effective decisions simply relying on a single decision maker [1-2]. More and more decision-making activities are dependent on joint involvement and determination of multiple decision makers. The shared decision-making of multi-stakeholders exists in various fields including large construction projects and public affairs management. Such group decision-making problems usually have the following three main features: (1) Multiple stakeholders are involved. Multiple decision makers will face complicated uncertainties derived from the event itself or internal and external environment during the decision making, then each decision maker can identify the categories and make judgments relatively clear for some alternatives, or familiar fields and focus areas; (2) Such group decision-making problems require cross-department, cross-industry and cross-discipline subjects to make group decisions. Due to the differences in physiology, psychology, behavior, background and culture, etc., different decision makers have different understandings, thoughts and judgments on the same problem, so the difference existing in evaluation results. A certain degree of antagonism or concession may exist in the process of decision making; (3) The final decision result depends on the evaluation of each decision maker. It is unknown whether there is any master-slave relation, dependency or ordering relation between multiple decision makers before the formation of an objective and reasonable evaluation result. That is, the relationship or weights of the so-called multiple decision makers are uncertain or vague. With respect to the group decision-making problem of multi-stakeholders on alternatives 
with category preference, it is urgent for group decision makers, for the purpose of development both in theory and practice to find out a decision-making method based on the correspondence between subjectivity and objectivity and seek for a satisfactory alternative with subjective concession and objective consistency for multiple subjects to coordinate the conflicts between multiple decision makers and help multiple decision makers head for the "dialogue" from "confrontation", provide a foundation for correct, scientific and reasonable group decision-making.

As for practice study, it is essentially an indication of information uncertainty and interest conflict when multiple stakeholders make decisions simultaneously. The applications of such problems include sustainable development problems of large infrastructures [3], E-Business risk evaluation [4], urban planning and filtration of schemes [5], supply chain selection and optimization [6], project selection of social industrial projects [7], etc. It can be seen that the game method is often used to study the decision-making of multi-stakeholders in terms of practice study. There are few quantitative researches of data. In terms of theoretical study, similar problems have gained much attention from scholars. Firstly, regarding the study on category preference information: (1) Focus on the study of preference information provided by decision makers for all alternatives (or evaluation objects) or some alternatives, for example, scholars have studied various methods of classification or sorting for alternatives in the field of multi-attribute decision-making according to the situations of comparison based on the reference [8], reference point and critical value based on different types of alternatives [9], strength and weakness relationship based on different alternative advantages [10], precise comparison [11] and fuzzy comparison [12] of different alternatives, etc.; (2) Focus on expression patterns of experts for different category preferences of alternatives, for example, regarding the situations where different decision makers have comparison information on alternatives [13], have different preference structure information [14], or have incomplete preference information on the result of group decision-making [15], the category judgments of different decision makers are similar to some extent [16], and multiple decision makers provide a certain level of preference for alternatives [17], etc., scholars have provided corresponding group decision-making methods. Secondly, regarding the study on weight determination of multiple stakeholders in group decision making: (1) Adopt the simplest and most direct method of subjective weight determination [18-19] which requires the decision makers know each other well or the interest relationship is relatively clear; (2) Adopt the method of objective weight determination [20-21]. The weight of the decision-making subject is determined through the preference information provided by the decision-maker. It should be mentioned that the weight determined by the method of objective weight determination is the dynamic weight of the decision makers which is often discussed in the academic circle; (3) Adopt the method of subjective and objective integrated weight determination [22-23] which is quite complex and requires the integration of subjective and objective information to correct the weight of the decision maker. In various similar decisionmaking theories and methods, many studies only discuss a single problem or individual analysis model or method. More often, values are assigned to the attribute weight or decision-maker weight beforehand in current studies. However, since the expertise, cognitive competence and practical experience of different decision makers are different in the circumstance of multiple decision makers, simply defining the attribute weight or decision-maker weight as known parameters in advance will greatly affect the result of group decision-making. It is questionable whether the result consists with the subjective category judgment of the decision-maker and conforms to objective requirements of group decision making.

This paper extracts a type of new group decision-making problem according to management practices: multiple decision makers have category preference for some 
alternatives and the issues of benefit restriction and unknown weights exist in these subjects, thus it boils down to the group decision-making problem of multi-stakeholders on alternatives with category preference. Optimize the attribute weight and the weight of each decision-maker by relevant principles of case-based learning method and fuzzy set under the category preference information respectively. Find out the master slave relation of multiple decision makers from group decision-making problems in the circumstance with interest relationship existing originally by meeting the subjective category preference information of different decision makers and objective facts of group decision-making in succession, and figure out a satisfactory method by which multiple decision makers can compromise, objectively reflect the relative importance of each attribute in each subject circumstance and the decision-making level of each decision-maker. Finally, verify the method with a specific case of decision making in urban planning. The study results have a certain theoretical value and practical significance for complicated multi-attribute group decision making.

\section{Problem Description}

\subsection{Problem Description and Thinking}

Generally, the typical, multi-person and multi-attribute decision-making issue is that different decision makers $e_{p}, p=1,2, \ldots, k$ rank the alternatives in accordance with a certain rule based on the given index $\omega_{j}, j=1,2, \ldots, m$ and the decision alternative $z_{i}, i=1,2, \ldots, n$. The multi-attribute classification decision is to classify alternatives into specific categories and the alternatives in the same category are of similar nature. In some decision-making cases, due to the great number of alternatives to be evaluated and the limitation of specialty or research interest, different decision makers often provide relatively definite category judgment information for some alternatives which can be called as judgment information for groups with category preference. These limited samples with relatively definite category information can be studied in line with the analysis method of case study, thus realizing ranking or classification of all alternatives based on conclusions of sample study. This method has a less strict requirement for the information extraction of decision markers and is easier for decision makers to submit and receive relevant information in their own specialties or their focus areas locally. Meanwhile, in the circumstance of multiple stakeholders, the levels of decision makers are not clear and the relation is vague, therefore the weight determination of multiple decision makers should fully reflect the decision-making levels of different decision makers which should not be treated in the same way.

On this basis, this paper describes the multi-attribute group decision-making on alternatives with category preference in the multi-stakeholders circumstance as below: $n$ alternatives form the alternative set $z=\left\{z_{i} \mid i=1,2, \ldots, n\right\} ; m$ attributes form the attribute set $W=\left\{\omega_{j} \mid j=1,2, \ldots, m\right\} ; k$ decision makers participate in decision-making and form the decision-making group $E=\left\{e_{p} \mid p=1,2, \ldots, k\right\}$. When the decision-maker $e_{p}$ makes a judgment, the weight vector of each attribute that he/she considers is $W^{p}=\left(\omega_{1}^{p}, \omega_{2}^{p}, \ldots \omega_{m}^{p}\right)$. For $\forall j \in\{1,2, \ldots, m\}, \omega_{j}^{p} \geq 0$ and $\sum_{j=1}^{m} \omega_{j}^{p}=1 ; \lambda_{p}$ is the weight of No. $p$ decision-maker, and the boundary of subject is unclear, that is, the weight vector $\lambda_{p}$ of each decision maker is unknown. For $\forall p \in\{1,2, \ldots, k\}, \lambda_{p} \geq 0, \sum_{p=1}^{k} \lambda_{p}=1 ; r_{i j}^{p}$ is the attribute judgment value given by No. $p$ decision-maker for No. $i$ alternative under No. $j$ index, without loss of 
generality. Assume that the attributes in $\mathrm{Z}$ are all very large (benefit type). Figure 1, shows the decision-making process of such problems.

Such problems have the following difficulties: (1) The preference information on alternatives from different decision makers is category preference information. A way should be found to balance the decision information and category preference information given by decision makers for all alternatives to determine whether the attribute weights consist with their subjective preferences or not; (2) Interest conflicts exist in difference decision makers, and the master slave relation is uncertain and quite vague. The method to use the uncertainty and vagueness of multiple decision makers should be developed to make the group judgment more comply with the requirements of objective facts.

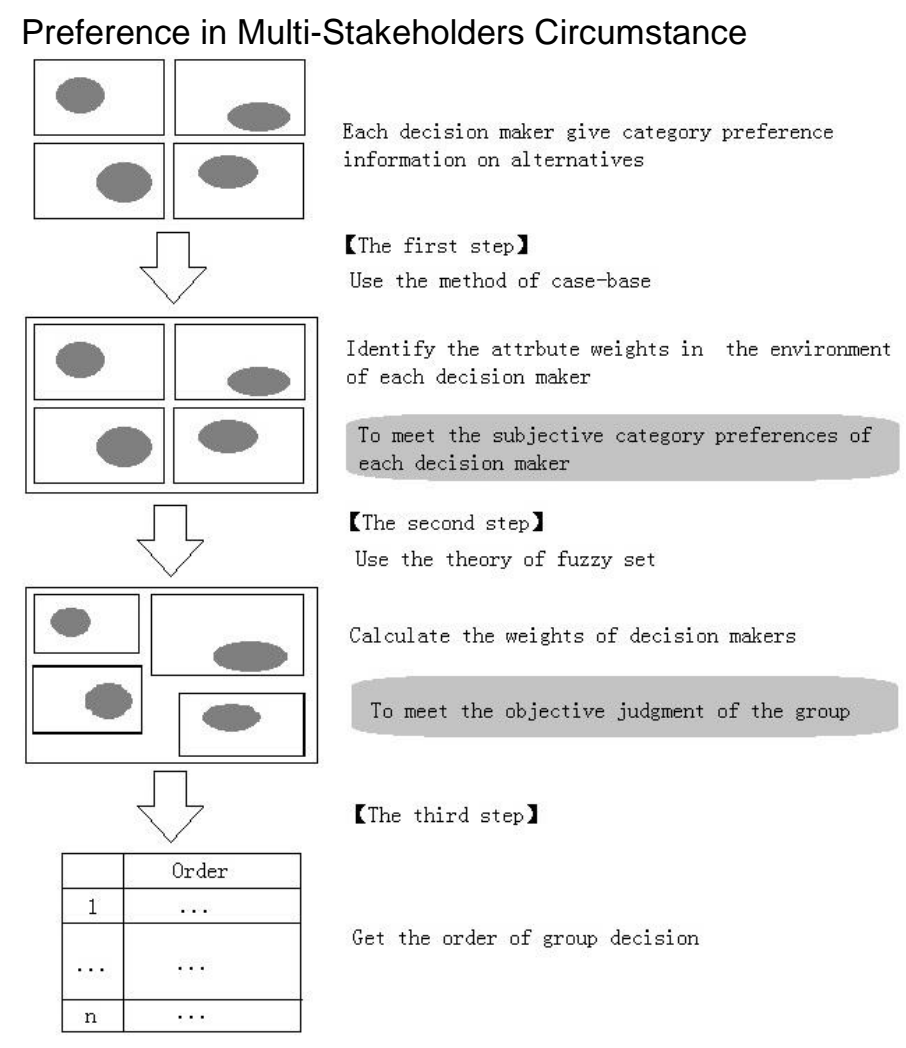

Figure 1. Group Decision-Making Process on Alternatives with Category

\subsection{Methods and Steps}

In order to realize the final ranking or classification of group decisions, the attribute weight in specific decision-maker circumstance and the weight of each decision-maker should be determined respectively. Since both weights are unknown, the method of casebased learning is first used in this paper to determine attribute weight vectors in different subject circumstances.

2.2.1. Learning Model of Attribute Weight Based on Category Preference Information: The grey target decision making has the principle of "non-uniqueness" in the grey system theory, reflecting the idea that the target is approachable, the plan can be improved and the approach can be optimized. The grey target decision making has been fully applied and embodied in the decision-making theory. This paper calculates the overall off-target distance of the alternative relative to positive and negative ideal points by the method of grey target decision making, and provides the case-based learning based 
on the category preference information, thus to obtain attribute weight vectors in different subject circumstances.

(1) Correction of positive and negative target centers and calculation of overall offtarget distance.

In grey target decision making, the selection of reference point is very critical. When positive and negative target centers are used for decision-making analysis, they should be set according to the features of the decision-making circumstance. In case of group decision-making, possible alternatives in the process of target center setting should be considered to set the positive and negative target centers of the determined group.

Definition 1: Assume that alternative $z_{i}^{p}$ is not in the decision alternative set of decision maker $e^{p}$, but may affect the existing evaluation plan or may occur in later comprehensive decisions, so the alternative is the potential alternative of the decision maker $e^{p}$.

After the analysis of potential alternative set according to Definition 1, the decision information of other decision makers should be considered fully during the setting of positive and negative target centers to consider the decision information provided by all decision makers comprehensively. The improvement is specifically as below:

Step 1: Set positive and negative target centers under the evaluation set of decision maker $e^{p}$. Assume that the positive target center and negative target center of the benefit type index set $r_{j}^{p}$ based on the existing alternative set of decision maker $e^{p}$ is $r_{0 j}^{p+}=\max \left\{r_{i j}^{p} \mid 1 \leq i \leq n, 1 \leq j \leq m\right\}$ and $r_{0 j}^{p-}=\min \left\{r_{i j}^{p} \mid 1 \leq i \leq n, 1 \leq j \leq m\right\}$ respectively.

Step 2: Find out the potential positive and negative target centers under the group evaluation set. According to the evaluation of all decision makers, analyze whether an individual decision maker is possible to have better or worse potential alternative set or not, and meanwhile, determine the potential positive and negative target centers under the evaluation set of each decision maker based on the suggestions given by all decision makers.

Assume that the positive target center and negative target center of the benefit type index set $a_{j}^{p *}$ based on the potential alternative set of the individual decision maker $e^{p}$ is $r_{j}^{p+^{*}} \quad$ and $r_{j}^{p-^{*}} \quad$ respectively, so $r_{j}^{p+^{*}}=\max \left\{r_{i j}^{p^{*}} \mid 1 \leq i \leq n^{*}, 1 \leq j \leq m\right\} \quad$ and $r_{j}^{p-*}=\min \left\{r_{i j}^{p^{*}} \mid 1 \leq i \leq n^{*}, 1 \leq j \leq m\right\}$.

The decision alternative set of a single decision makers and alternative sets of all decision makers form all possible decision alternatives. If there is no potential alternative set for a single decision maker $e^{p}$, the positive target center $r_{j}^{+}$and negative target center $r_{j}^{-}$of the decision maker $e^{p}$ are $r_{0 j}^{p+}$ and $r_{0 j}^{p-}$ respectively; if there is potential alternative set for a single decision maker $e^{p}$, the corrected positive target center and negative target center should be $r_{j}^{+}=\max \left(r_{0 j}^{p+}, r_{j}^{p++^{+}}\right)$and $r_{j}^{-}=\min \left(r_{0 j}^{p-}, r_{j}^{p-{ }^{-}}\right)$respectively.

Definition 2: Assuming $r_{j}^{p+}=\max \left\{r_{i j}^{p} \mid 1 \leq i \leq n, 1 \leq j \leq m\right\}, r_{j}^{p+}=\left\{r_{1}^{p+}, r_{2}^{p+}, \ldots, r_{m}^{p+}\right\}$ is called as the optimal effect vector of grey target decision making, representing the positive target center of the decision matrix of subject $p$ under attribute $j$. Assuming $r_{j}^{p-}=\min \left\{r_{i j}^{p} \mid 1 \leq i \leq n, 1 \leq j \leq m\right\}, r_{j}^{p-}=\left\{r_{1}^{p-}, r_{2}^{p-}, \ldots, r_{m}^{p-}\right\}$ is called as the worst effect vector of grey target decision making, representing the negative target center of the decision matrix of subject $p$ under attribute $j$.

The corresponding positive off-target distance, negative off-target distance and positive and negative off-target distances are as below: 
Definition 3: The positive off-target distance of the decision-making subject $p$ for alternative $z_{i}$ is:

$$
\varepsilon_{i}^{p+}=\omega_{1} d\left(r_{i 1}^{p}, r_{1}^{p+}\right)+\omega_{2} d\left(r_{i 2}^{p}, r_{2}^{p+}\right)+\ldots+\omega_{m} d\left(r_{i m}^{p}, r_{m}^{p+}\right)=\sum_{j=1}^{m} \omega_{j} d\left(r_{i j}^{p}, r_{j}^{p+}\right)
$$

The negative off-target distance of the decision-making subject $p$ for alternative $z_{i}$ is:

$\varepsilon_{i}^{p-}=\omega_{1} d\left(r_{i 1}^{p}, r_{1}^{p-}\right)+\omega_{2} d\left(r_{i 2}^{p}, r_{2}^{p-}\right)+\ldots+\omega_{m} d\left(r_{i m}^{p}, r_{m}^{p-}\right)=\sum_{j=1}^{m} \omega_{j} d\left(r_{i j}^{p}, r_{j}^{p-}\right)$

The distance between positive target center and negative target center of the decisionmaking subject $p$ for alternative $z_{i}$ is:

$$
\varepsilon_{i}^{p 0}=\omega_{1} d\left(r_{1}^{p+}, r_{1}^{p-}\right)+\omega_{2} d\left(r_{2}^{p+}, r_{2}^{p-}\right)+\ldots+\omega_{m} d\left(r_{m}^{p+}, r_{m}^{p-}\right)=\sum_{j=1}^{m} \omega_{j} d\left(r_{j}^{p+}-r_{j}^{p-}\right)
$$

In reference [24], the projection of the connecting line between positive target center and negative target center of decision alternative is defined as the overall off-target distance, therefore:

Definition 4: The projection of the connecting line between positive target center and negative target center of decision alternative $z_{i}$ is defined as the overall off-target distance of the decision-making subject $p$ for the alternative $z_{i}$, as below:

$$
\varepsilon_{i}^{\mathrm{p}^{*}}=\frac{\left(\varepsilon_{i}^{p+}\right)^{2}+\left(\varepsilon_{i}^{p 0}\right)^{2}-\left(\varepsilon_{i}^{p-}\right)^{2}}{2 \varepsilon_{i}^{p 0}}
$$

The overall off-target distance here shows the quality of the effect vector: The smaller the overall off-target distance of $z_{i}$, the better the decision alternative will be; the larger the overall off-target distance of $z_{i}$, the worse the decision alternative will be.

According to reference [24], the distance $\varepsilon_{i}^{0}$ between positive target center and negative target center of each alternative is a constant, so the formula above can be changed into:

$$
\varepsilon_{i}^{p}=\left(\varepsilon_{i}^{p+}\right)^{2}+\left(\varepsilon_{i}^{p 0}\right)^{2}-\left(\varepsilon_{i}^{p-}\right)^{2}
$$

(2) Determination method of attribute weight based on case study.

For No. $i$ decision alternative under the judgment of decision-making subject $p$, it is required to adjust the weight $\omega_{j}^{p}(j=1,2, \ldots, m)$ to minimize the overall off-target distance, expressed as below:

$$
\begin{aligned}
& \min \varepsilon^{p}=\min \left(\varepsilon_{1}^{p}, \varepsilon_{2}^{p}, \ldots, \varepsilon_{n}^{p}\right) \\
& \text { s.t. } \sum_{i=1}^{m} \omega_{j}^{p}=1, \omega_{j}^{p} \geq 0, j=1,2, \ldots, m
\end{aligned}
$$

In consideration of the fair competition between all alternatives, the following expression can be obtained according to Expression (5):

$$
\min \beta^{p}=\min \sum_{i=1}^{n} \varepsilon_{i}^{p}=\min \sum_{i=1}^{n} \sum_{j=1}^{m} \omega_{j}{ }^{2} d\left(r_{i j}^{p}, r_{j}^{p+}\right)+\sum_{i=1}^{n} \sum_{j=1}^{m} \omega_{j}{ }_{j}^{2} d\left(r_{i j}^{p+}, r_{j}^{p-}\right)-\sum_{i=1}^{n} \sum_{j=1}^{m} \omega_{j}{ }^{2} d\left(r_{i j}^{p}, r_{j}^{p-}\right)
$$

Definition 5: If a decision maker $e_{p}$ believes $l(l<n)$ alternatives in alternative set $Z=\left\{z_{i} \mid i=1,2, \ldots, n\right\}$ are similarly excellent and should be regarded as a equivalent 
category, these alternatives of the equivalent category deemed by the decision maker $e_{p}$ is recorded as $Z^{p \#}=\left\{z_{i}^{p \#} \mid i=1,2, \ldots, l\right\}\left(Z^{p \#} \subset Z\right)$, so its relation can be expressed as $z_{1}^{p \#} \approx z_{2}^{p \#} \approx \ldots \approx z_{1}^{p \#}$, namely, the overall off-target distance of any two alternatives in alternative set $Z^{p \#}$ is equivalent, and the relation can be expressed as $\varepsilon_{1}^{p \#} \approx \varepsilon_{2}^{p \#} \approx \ldots \approx \varepsilon_{i}^{p \#}$. For any two alternatives $z_{i}^{p \#}$ and $z_{i+1}^{p \#}$ therein, to make $\varepsilon_{i}^{p \#} \approx \varepsilon_{i+1}^{p \#}, \frac{\varepsilon_{i}^{p \#}}{\varepsilon_{i+1}^{p \#}} \approx 1$ is required. Introduce the fluctuating variable $0 \leq \rho \leq 1$. So $\frac{\varepsilon_{i}^{p \#}}{\varepsilon_{i+1}^{p \#}}$ can fluctuate in a certain range as below: $\rho \leq \frac{\varepsilon_{i}^{p \#}}{\varepsilon_{i+1}^{p \#}} \leq \frac{1}{\rho}$, on this basis, the following expression can be obtained:

$\left\{\begin{array}{l}\rho \leq \frac{\varepsilon_{i}^{p \#}}{\varepsilon_{i+1}^{p \#}} \leq \frac{1}{\rho} \\ i=1,2, \ldots, l-1, z_{i}^{p \#} \in Z^{P \#}, Z^{P \#} \subset Z\end{array}\right.$

Regarding the value of $\rho, \rho$ reflects the equivalence of the overall off-target distance of any two alternatives in alternative set $Z^{P \#}$, namely the similarity precision of any two alternatives in alternative set $Z^{P \#}$ : when $\rho$ increases, the similarity precision of any two alternatives in $Z^{P \#}$ becomes higher, that is, they are more similar; when $\rho$ decreases, the similarity precision of any two alternatives in $Z^{P \#}$ becomes lower, that is, they are less similar; especially, when $\rho=1$, there is no difference for two alternatives in $Z^{p \#}$ in terms of excellence, that is, they are exactly the same in terms of excellence.

If the category preference given by decision-maker $p$ includes the category preference mentioned in Definition 5, a linear programming model (M-1) can be established:

$$
\begin{aligned}
& \min \beta^{p}=\min \sum_{i=1}^{n} \varepsilon_{i}^{p} \\
& \text { s.t. }\left\{\begin{array}{l}
\rho \leq \frac{\varepsilon_{i}^{p \#}}{\varepsilon_{i+1}^{p \#}} \leq \frac{1}{\rho} \\
i=1,2, \ldots, l-1, z_{i}^{p \#} \in Z^{P \#}, Z^{P \#} \subset Z
\end{array}\right.
\end{aligned}
$$

According to Expression (1) - (5), the above objective programming model can be converted into (M-2):

$$
\begin{aligned}
& \min \beta=\min \sum_{i=1}^{n} \sum_{j=1}^{m} \omega_{j}^{2} d\left(r_{i j}^{p}, r_{j}^{p+}\right)+\sum_{i=1}^{n} \sum_{j=1}^{m} \omega_{j}^{2} d\left(r_{i j}^{p+}, r_{j}^{p-}\right)-\sum_{i=1}^{n} \sum_{j=1}^{m} \omega_{j}^{2} d\left(r_{i j}^{p}, r_{j}^{p-}\right) \\
& \rho . t .\left\{\begin{array}{c}
\sum_{j=1}^{m} \omega_{j}^{2} d\left(r_{i j}^{p \#}, r_{j}^{p+}\right)+\sum_{j=1}^{m} \omega_{j}^{2} d\left(r_{j}^{p+}, r_{j}^{p-}\right)-\sum_{j=1}^{m} \omega_{j}^{2} d\left(r_{i j}^{p \#}, r_{j}^{q_{j}-}\right) \\
\sum_{j=1}^{m} \omega_{j}^{2} d\left(r_{(i+1) j}^{p \#}, r_{j}^{p+}\right)+\sum_{j=1}^{m} \omega_{j}^{2} d\left(r_{j}^{p+}, r_{j}^{p-}\right)-\sum_{j=1}^{m} \omega_{j}^{2} d\left(r_{(i+1) j}^{p \#}, r_{j}^{p-}\right)
\end{array} \frac{1}{\rho}\right. \\
& i=1,2, \ldots, l-1, z_{i}^{p \#} \in Z^{P \#}, Z^{P \#} \subset Z, \sum_{i=1}^{m} \omega_{j}^{p}=1, \omega_{j}^{p} \geq 0, j=1,2, \ldots, m, i=1,2, \ldots, n
\end{aligned}
$$

Theorem 1: Model M-2 must have the optimal solution.

Demonstration: The feasible region of Model M-2 is bounded convex hull, and the objective functions are continuous and have the maximum $\left(\omega_{j}<1\right)$. As the objective functions are continuous functions with the maximum, the feasible region with bounded 
convex hull must have the optimal solution, and therefore Model M-2 must have the optimum solution.

Solve the above model through software lingo11.0, and the attribute weight vectors in different subject circumstances can be obtained: $W^{p}=\left(\omega_{1}^{p}, \omega_{2}^{p}, \ldots \omega_{m}^{p}\right)$

2.2.2. Multi-Stakeholders Weight Optimization Method: In the multi-stakeholders circumstance, different decision makers often consider lots of factors from the perspective of their own interests in the process of decision making. Since their expertise, cognitive competence and practical experience, etc. are different and the relationship therein is complicated, the weight determination of decision-making subjects, in the circumstance of multiple decision-maker, should fully reflect the decision-making levels and interests of decision makers which should not be treated in the same way. For these reasons, the weight determination is also of high uncertainty and vagueness. Based on the features of such fuzzy information, a method of multiple decision-maker weight assignment based on the fuzzy set theory is provided below.

In fact, the calculation process of the decision-maker weight can be seen as a special form of reference [25]. According to reference [25], when fuzzy information is used, all information during the decision making should be fully used. With all available information, form a fuzzy but relative consistent opinion by using triangular fuzzy numbers. In this paper, the method of scoring is used for assignment of each alternative, and later the minimum, mean and maximum from the scores are used as three measurements of triangular fuzzy numbers. In this study, as the attribute weight in each subject circumstance has been obtained through the steps in 2.2.1, it is more reasonable and scientific to represent the scoring of each alternative through the overall off-target distance of each alternative in each subject circumstance. Therefore, the application in reference [25] is improved in this paper: take the minimum overall off-target distance, maximum overall off-target distance and mean overall off-target distance in each subject circumstance as three measurements of triangular fuzzy numbers. This method uses the calculation result based on the subject category preference information in 2.2.1 effectively and shows more respect for the subjective evaluation of the decision maker than the method of direct scoring. And it is easier for multiple decision makers to receive the final result.

Convert the overall off-target distance $\varepsilon_{i}^{p}$ of alternative $z_{i}$ for decision maker ${ }^{e}$ into the triangular fuzzy number:

$\tilde{\varepsilon_{i}^{p}}=\left(\varepsilon_{1}^{p}, \varepsilon_{2}^{p}, \varepsilon_{3}^{p}\right)=\left\{\left[\min \left(\varepsilon_{1}^{p}, \varepsilon_{2}^{p} \ldots \varepsilon_{n}^{p}\right)\right],\left[\sum_{i=1}^{m} \varepsilon_{i}^{p} / n\right],\left[\max \left(\varepsilon_{1}^{p}, \varepsilon_{2}^{p} \ldots \varepsilon_{n}^{p}\right)\right]\right\}$

Where $\varepsilon_{1}^{p}$ is the overall off-target distance of the worst alternative thought by the decision maker $e_{p}$ which represents pessimistic estimate; $\varepsilon_{3}^{p}$ is the overall off-target distance of the optimal alternative thought by the decision maker $e_{p}$ which represents optimistic estimate; $\varepsilon_{2}^{p}$ is the mean overall off-target distance of all alternatives thought by the decision maker $e_{p}$. According to relevant demonstrations in reference [25], the objective weight assignment of uncertain multi-subject should be as below:

$\lambda=\left(B^{-1} I\right) / I^{T} B^{-1} I$

Where $\lambda=\left(\lambda_{1}, \lambda_{2}, \ldots, \lambda_{k}\right)^{T}$ is the weight of each subject, $I=(1,1, \ldots, 1)^{T}$, and the matrix B is as below: 


$$
B=\left(b_{i j}\right)_{k \times k}=\left\{\begin{array}{cccc}
(k-1)\left(\sum_{l=1}^{3} \varepsilon_{1 l}^{2}\right) & -\sum_{l=1}^{3} \varepsilon_{1 l} \varepsilon_{2 l} & \ldots & -\sum_{l=1}^{3} \varepsilon_{1 l} \varepsilon_{k l} \\
-\sum_{l=1}^{3} \varepsilon_{2 l} \varepsilon_{1 l} & (k-1)\left(\sum_{l=1}^{3} \varepsilon_{2 l}^{2}\right) & \ldots & -\sum_{l=1}^{3} \varepsilon_{2 l} \varepsilon_{k l} \\
\ldots & \ldots & \ldots & \ldots \\
-\sum_{l=1}^{3} \varepsilon_{k l} \varepsilon_{1 l} & -\sum_{l=1}^{3} \varepsilon_{k l} \varepsilon_{2 l} & \ldots & (k-1)\left(\sum_{l=1}^{3} \varepsilon_{k l}^{2}\right)
\end{array}\right]
$$

Therefore, the weights of different decision makers can be obtained. Since the data source of the method is the category judgment information of each subject, the weights of decision makers obtained have the following three features: (1) They can be regarded as the weight solutions received or compromised by each subject on the basis of the category judgment information of the decision maker, thus avoiding many unfavorable factors of subjective weighting; (2) In multi-stakeholders circumstance, they can be considered as the comprehensive reflection of interests of different subjects and have objectivity and acceptability; (3) They can be seen as the basis of correctness or reliability for various decision maker category judgment information, thus avoiding some inherent judgment biases that may exist in group decision making (for example, the weight calculation result may be contrary to previous weight judgment result which has been embodied in the method comparison in the case part of this paper).

2.2.3. Group Preference Aggregation in Multi-Stakeholders Circumstance: Take the overall off-target distance of each alternative as the objective function of such problem of group decision-making in multiple decision maker circumstance, and apply the method of linear weighting, then the overall off-target distance of each alternative ${ }^{z_{i}}$ in case of group decision-making can be obtained:

$\varepsilon\left(z_{i}\right)=\sum_{p=1}^{k} \lambda_{p} \varepsilon_{i}^{p}=\sum_{p=1}^{k} \lambda_{p}\left(\left(\varepsilon_{i}^{p+}\right)^{2}+\left(\varepsilon_{i}^{p 0}\right)^{2}-\left(\varepsilon_{i}^{p-}\right)^{2}\right)$

As the weight vector $W^{p}=\left(\omega_{1}^{p}, \omega_{2}^{p}, \ldots \omega_{m}^{p}\right)$ under each decision maker and the weight ${ }^{\lambda_{p}}$ of the decision-making expert are all known, with Model M-2 and Expression (6), the total order relation of the alternative set can be established by using Expression (7).

Therefore, the steps of the method in this paper can be summarized as follows:

Step 1: Correct the positive and negative target centers of the group according to the method in 2.2.1 (1), and calculate the distance of each alternative to the positive and negative target centers of the group. Calculate the attribute weight in each decision maker circumstance according to 2.2.1 (2).

Step 2: Determine the weight of each decision maker according to 2.2.2, and judge the category preference information credibility of the subject based on the result.

Step 3: Calculate the overall off-target distance of each alternative in the circumstance of group decision-making according to 2.2.3, and figure out the total order relation of the alternative according to the overall off-target distance.

\section{Case}

Urban planning issue is one of typical issues in public management affairs. The evaluation indexes of urban planning generally include: environment level $\mathrm{w}_{1}$, economic development level $\mathrm{w}_{2}$, cultural construction level $\mathrm{w}_{3}$, ROI level $\mathrm{w}_{4}$ and traffic system index level $\mathrm{w}_{5}$. Generally, the government agencies $e^{1}$, experts in urban planning $e^{2}$, constructor representatives $e^{3}$ and the public $e^{4}$ participated in the investigation and 
practice on behalf of multiple decision makers before the planning. The four decision makers represent the interests of respective party, with unclear boundary. The four decision makers offered evaluation information (already normalized, as shown in Table 1) on relevant indexes in their field of view for alternative $z_{1}-z_{5}$ respectively, and meanwhile, different decision-making subjects provided their own category preference information, as shown in the grey part of Table 1. Determine the weight according to the alternatives in this paper, and rank the alternatives.

Table 1. Attribute Values and Category Preference Information Provided by Multiple Subjects

\begin{tabular}{|c|c|c|c|c|c|c|c|c|c|c|c|}
\hline program & $\mathrm{w}_{1}$ & $\mathrm{~W}_{2}$ & $\mathrm{w}_{3}$ & $\mathrm{~W}_{4}$ & $\mathrm{~W}_{5}$ & program & $\mathrm{W}_{1}$ & $\mathrm{~W}_{2}$ & $\mathrm{w}_{3}$ & $\mathrm{~W}_{4}$ & $\mathrm{~W}_{5}$ \\
\hline \multicolumn{6}{|c|}{$\begin{array}{l}\text { Decision maker } e^{1} \text { gave a property value and } \\
\text { category preference }\end{array}$} & \multicolumn{6}{|c|}{$\begin{array}{c}\text { Decision maker } e^{2} \text { gave a property value and } \\
\text { category preference }\end{array}$} \\
\hline $\mathrm{Z}_{1}{ }^{1}$ & 0.9675 & 0.3130 & 0.3575 & 0.9581 & 0.2177 & $\mathrm{z}_{1}^{2}$ & 1 & 0.5340 & 0.4894 & 0.9881 & 0.2348 \\
\hline $\mathrm{Z}_{2}{ }^{1}$ & 0.6836 & 1 & 1 & 0.3336 & 1 & $\mathrm{z}_{2}{ }^{2}$ & 0.6762 & 0.9156 & 0.8775 & 0.4352 & 1 \\
\hline $\mathrm{Z}_{3}{ }^{1}$ & 0.4887 & 0.4957 & 0.3944 & 0.6905 & 0.3864 & $\mathrm{z}_{3}{ }^{2}$ & 0.4602 & 0.1614 & 0.1861 & 0.7500 & 0.1579 \\
\hline $\mathrm{Z}_{4}{ }^{1}$ & 0.6741 & 0.3457 & 0.2053 & 1 & 0 & $\mathrm{z}_{4}{ }^{2}$ & 0.8971 & 0.3362 & 0.2151 & 1 & 0 \\
\hline $\mathrm{Z}_{5}{ }^{1}$ & 0.0872 & 0.3652 & 0.0443 & 0.3600 & 0.4737 & $\mathrm{z}_{5}^{2}$ & 0.0450 & 0.0913 & 0.0679 & 0.2157 & 0.1447 \\
\hline $\mathrm{Z}_{6}{ }^{1}$ & 0 & 0 & 0.3752 & 0 & 0.4617 & $\mathrm{z}_{6}{ }^{2}$ & 0.1224 & 0 & 0.5784 & 0.1003 & 0.3247 \\
\hline \multicolumn{6}{|c|}{$\begin{array}{l}\text { Decision maker } e^{3} \text { gave a property value and } \\
\text { category preference }\end{array}$} & \multicolumn{6}{|c|}{$\begin{array}{c}\text { Decision maker } e^{4} \text { gave a property value and } \\
\text { category preference }\end{array}$} \\
\hline $\mathrm{z}_{1}^{3}$ & 1 & 0.6989 & 0.5820 & 0.6827 & 0.6534 & $\mathrm{z}_{1}^{4}$ & 1 & 0.4251 & 0.4675 & 0.7889 & 0.8466 \\
\hline $\mathrm{z}_{2}^{3}$ & 0.5807 & 0.1858 & 0.2260 & 0.4306 & 0.1232 & $\mathrm{z}_{2}^{4}$ & 0.6758 & 1 & 1 & 0.3026 & 1 \\
\hline $\mathrm{z}_{3}{ }^{3}$ & 0.4087 & 0.4946 & 0.3748 & 0.5704 & 0.3879 & $\mathrm{z}_{3}{ }^{4}$ & 0.5789 & 0.6857 & 0.5784 & 0.385 & 0.5794 \\
\hline $\mathrm{z}_{4}{ }^{3}$ & 0.1785 & 0.0783 & 0.1551 & 0.4372 & 0.2105 & $\mathrm{z}_{4}{ }^{4}$ & 0.1299 & 0.2989 & 0.0384 & 0.6633 & 0.2919 \\
\hline $\mathrm{z}_{5}^{3}$ & 0.1946 & 0.0293 & 0 & 0.3313 & 0.1962 & $\mathrm{z}_{5}{ }^{4}$ & 0.2441 & 0.3761 & 0.2999 & 0.3871 & 0.4821 \\
\hline $\mathrm{z}_{6}{ }^{3}$ & 0 & 0 & 0.3875 & 0 & 0.4789 & $\mathrm{Z}_{6}{ }^{4}$ & 0.1427 & 0.0293 & 0.1388 & 0.4065 & 0.4103 \\
\hline
\end{tabular}

Step 1: Correct the positive and negative target centers of the group, $r_{0 j}^{(1-4)+}=\{1,1,1,1,1\}$, $r_{0 j}^{(1-4)-}=\{0,0,0,0,0\}$, and calculate the distance from the attribute value to the positive and negative target centers of each alternative under different attributes.

Use the method in 2.2.1 (2), take $\rho=0.95$, and solve the attribute weight vectors on alternatives with category preference information for all subjects, respectively as below:

Decision-making subject $e^{1}: \omega^{1}=(0.0549,0.3567,0.1108,0.3157,0.1619)$;

Decision-making subject $e^{2}: \omega^{2}=(0.2543,0.0990,0.0956,0.0970,0.4541) ;$

Decision-making subject $e^{3}: \omega^{3}=(0.4355,0.0903,0.0421,0.0660,0.3661)$;

Decision-making subject $e^{4}: \omega^{4}=(0.1215,0.1503,0.1074,0.2288,0.3920) ;$

Step 2: Calculate the vector of overall off-target distance $\varepsilon_{i}^{p}$ of each alternative $z_{i}$ in the circumstance of each decision-making subject $e_{p}$ by using the method in 2.2.2, thus to convert the overall off-target distance $\varepsilon_{i}^{p}$ of the decision maker $e_{p}$ on alternative $z_{i}$ into corresponding triangular fuzzy number, as below:

$$
\begin{aligned}
& {\tilde{\varepsilon_{i}}}^{1}=(0.1347,0.2843,0.1347) \\
& \tilde{\varepsilon}_{i}^{2}=(0.0564,0.3765,0.5259) \\
& \tilde{\varepsilon}_{i}^{3}=(0.1021,0.4264,0.5462) \\
& \tilde{\varepsilon}_{i}^{4}=(0.0826,0.2292,0.3324)
\end{aligned}
$$

Matrix B: 


$$
\begin{aligned}
& B=\left(b_{i j}\right)_{4 \times 4}=\left[\begin{array}{cccc}
2.4793 & -0.8523 & -0.81121 & -0.89407 \\
-0.8523 & 2.6599 & -0.84575 & -0.91821 \\
-0.81121 & -0.84575 & 2.4386 & -0.88532 \\
-0.89407 & -0.91821 & -0.88532 & 2.9876
\end{array}\right], \\
& B^{-1}=\left(b_{i j}\right)_{4 \times 4}=\left[\begin{array}{llll}
8.7027 & 8.1060 & 8.4671 & 7.6047 \\
8.1060 & 8.1047 & 8.1714 & 7.3381 \\
8.4671 & 8.1714 & 8.8438 & 7.6660 \\
7.6047 & 7.3381 & 7.6660 & 7.1375
\end{array}\right]
\end{aligned}
$$

According to Expression (6), the weight vector of the decision maker can be obtained: $\lambda=(0.2579,0.2489,0.2600,0.2332)$. Namely, the weight of government agencies $e^{1}$ is 0.2579 ; the weight of experts in urban planning $e^{2}$ is 0.2489 ; the weight of constructor representatives $e^{3}$ is 0.2600; and the weight of the public $e^{4}$ is 0.2332 .

Step 3: Calculate the overall off-target distance of each alternative in multiple decision maker circumstance according to 2.2.3, and obtain the optimum ranking of each alternative in circumstance of each decision maker and the ranking of the group as shown in Table 2.

\section{Table 2. Utility Values and Ranking of Alternatives in the Field of View of Each Decision Maker, Final Group Utility Values and Ranking of Alternatives}

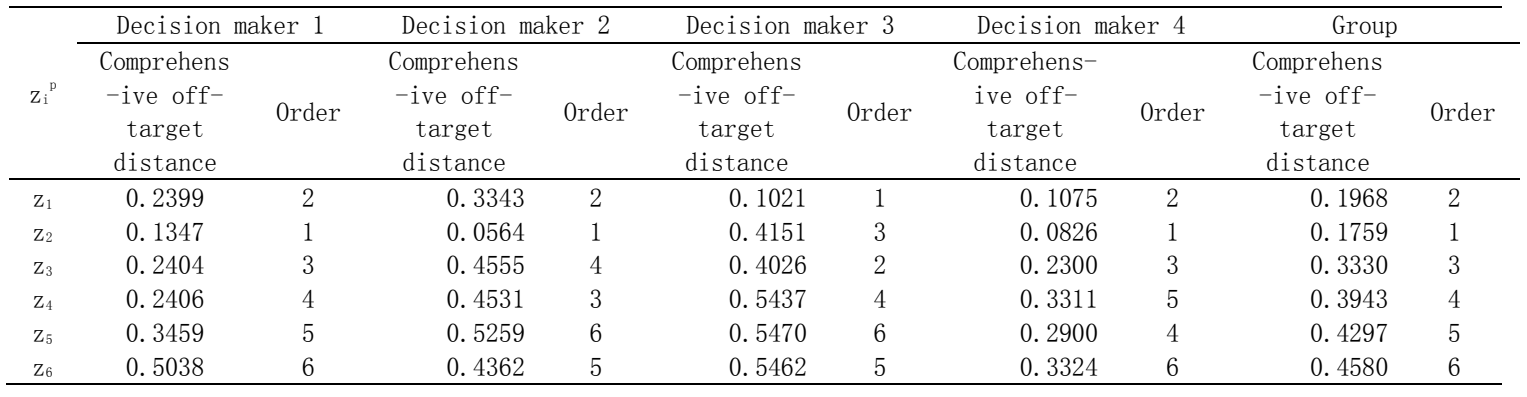

\section{Method Comparison:}

This paper has two main innovations: 1 . The category preferences of multiple decision makers on alternatives are specially considered to obtain the attribute weight of subjective judgment for different decision makers through calculation; 2 . The weight information of multiple subjects are determined by the method of fuzzy mathematics to determine the weight information of multiple decision makers. In this respect, this paper takes references [8-10] for the method comparison of the first innovation and takes intuitive judgment information and reference [22] for the method comparison of the second innovation.

1. Method comparison in attribute weights (For simplicity and intuitiveness, compare the utility values). To compare the method with that in reference [8], take alternatives in the same category indicating "slightly better". For the ranking results, see Table 3, (reference [8] sequence) and the comparison between calculated utility values of decision maker No.1 (Figure 2). According to the calculation results, the ranking conditions are basically the same, but according to the utility value results, the utility value difference between alternatives in the same category and alternatives in different categories is less significant than that in this paper (for example, the data of decision maker No.1 are compared in Figure 2. From the manifestation, the utility value difference between alternatives in the same category and alternatives in different categories in the method of this paper is greater). To compare the method with that in reference [10], determine the 
order relation for alternatives in the same category as $\approx$, assume other corresponding alternative order relations as $>$ and $\prec$ in turn, and make decisions based on the correlation of ordinal information. For the ranking results, see

Table 3, (reference [10] sequence) and the comparison between calculated utility values of decision maker No.1 (Figure 3). According to the calculation results, the ranking conditions are basically the same, but according to the utility value results, the similarity of alternatives in the same category is less significant than that in this paper (for example, the data of decision makers No.1 are compared in Figure 3. From the manifestation, alternative 1 , alternative 3 and alternative 4 of the same category in the method of this paper are less volatile).

Table 3. Utility Values and Ranking of Alternatives in this Paper and Reference [8] and [10]

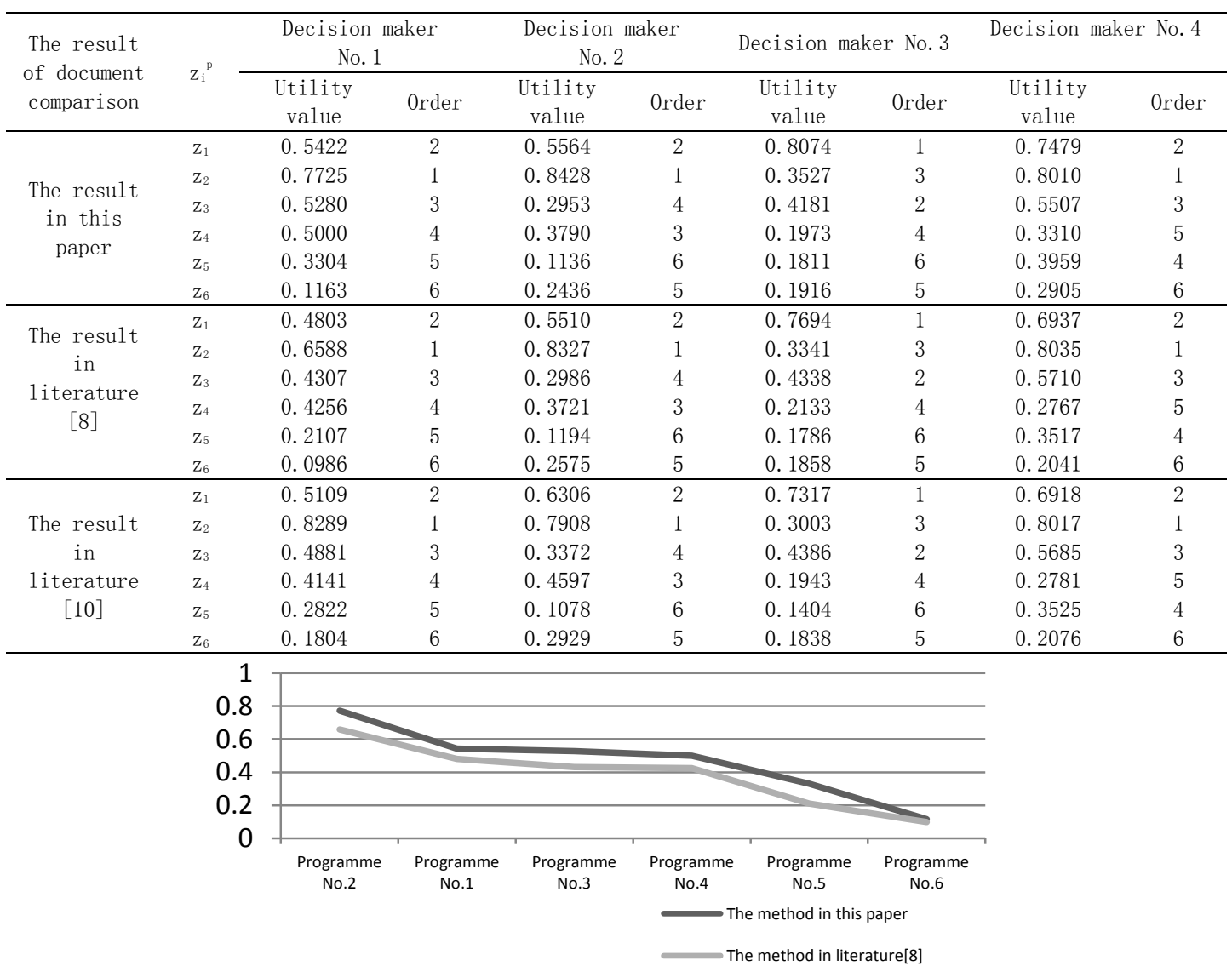

Figure 2. Comparison of Utility Values for Each Alternative between this Paper and Literature[8] (with Decision Maker No. 1 As An Example)

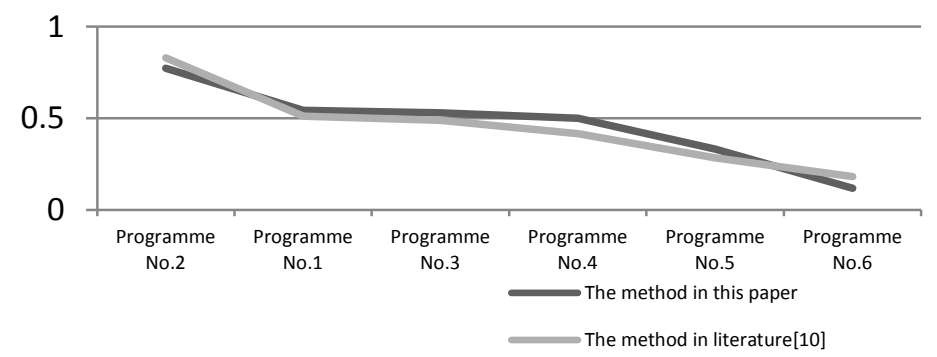

Figure 3. Comparison of Utility Values for Each Alternative between this Paper and Literature[10] (with Decision Maker No.1 as an Example) 
2. Method comparison in weights of decision makers.

(1) In this paper, the objective weighting result in the method is different from previous intuitive judgment of people. People thought that government agencies should take up more subject weight during the decision making and that experts in urban planning as the "neutral" party in terms of interests should make objective judgments. However, according to the calculation result, the category preference judgment information of the constructor may be more correct and reasonable, thus the subject weight of the constructor is higher. From the perspective of the respect for the credibility of subjective preference judgment information, we should respect the calculation method on the decision-making subject weight in this paper more.

(2) The main idea in reference [22] is to assign the weight at each evaluation index for each expert subject by using the subject weighting first, and then add the adjusted values of objective weight vectors. For the fairness of comparison, the subjective weight at each evaluation index for different decision-making subjects should be assigned with the same value, namely, the subjective weight vectors at 5 evaluation indexes for 4 decision makers are all $\lambda^{*}=(0.25,0.25,0.25,0.25)$. Calculate the objective weight vectors at the evaluation indexes for 4 decision makers. Through the coefficient adjustment, the comprehensive weight vectors at 5 evaluation indexes for 4 decision makers are finally obtained:

$\lambda^{\omega 1}=(0.2064,0.2948,0.1964,0.3024)$

$\lambda^{\omega 2}=(0.1996,0.2721,0.2324,0.2959)$

$\lambda^{\omega 3}=(0.2784,0.1916,0.2788,0.2512)$

$\lambda^{\omega 4}=(0.2574,0.3011,0.1236,0.3179)$

$\lambda^{\omega 5}=(0.2667,0.2012,0.1789,0.3532)$

Thus, the decision makers' evaluations for 6 alternatives at various indexes are obtained. Assuming that the weights for 5 evaluation indexes are set as $\omega=(0.2,0.2,0.2,0.2,0.2)$, the total score of each alternative can be obtained (as shown in Table 4). According to the ranking results, two methods are the same, which indicates that the weight assignment of multiple decision makers in the method of this paper is basically reasonable. The weight of the decision maker can be obtained directly through the method in this paper, so, in this respect, it is better than that in reference [22].

Table 4. Evaluation of Each Attribute and Total Score of Each Alternative Calculated Through the Method in Reference [22]

\begin{tabular}{|c|c|c|c|c|c|c|}
\hline$w_{j}$ & $\begin{array}{c}\text { Program } \\
\text { No. } 1 \\
\end{array}$ & $\begin{array}{c}\text { Program } \\
\text { No. } 2 \\
\end{array}$ & $\begin{array}{c}\text { Program } \\
\text { No. } 3 \\
\end{array}$ & $\begin{array}{c}\text { Program } \\
\text { No. } 4 \\
\end{array}$ & $\begin{array}{c}\text { Program } \\
\text { No. } 5 \\
\end{array}$ & $\begin{array}{c}\text { Program } \\
\text { No. } 6 \\
\end{array}$ \\
\hline $\mathrm{w}_{1}$ & 0.9933 & 0.6586 & 0.4919 & 0.4779 & 0.1433 & 0.0792 \\
\hline $\mathrm{W}_{2}$ & 0.4960 & 0.7878 & 0.4607 & 0.2671 & 0.2158 & 0.0087 \\
\hline $\mathrm{w}_{3}$ & 0.4729 & 0.7607 & 0.3952 & 0.1540 & 0.1007 & 0.3582 \\
\hline $\mathrm{w}_{4}$ & 0.8793 & 0.3663 & 0.5965 & 0.8234 & 0.3216 & 0.1594 \\
\hline $\mathrm{W}_{5}$ & 0.5212 & 0.8431 & 0.4089 & 0.1408 & 0.3608 & 0.4191 \\
\hline $\begin{array}{c}\text { The total } \\
\text { result of each } \\
\text { program }\end{array}$ & 0.6725 & 0.6834 & 0.4706 & 0.3727 & 0.2285 & 0.2049 \\
\hline Order & 2 & 1 & 3 & 4 & 5 & 6 \\
\hline
\end{tabular}

\section{Conclusion}

Firstly, the category preference information of the decision maker, representing the "intuitive judgment" of the subject during the decision making, is the judgment information on alternatives of different decision makers in their own professional backgrounds within the field of view. The attribute weight reflects the importance of 
different attributes in the formation of decisions. Therefore, the subjective judgment information or intuitive judgment information given by existing decision makers should be fully used and learnt in the determination of attribute weight. Secondly, the weight of the decision maker represents the decision-making power of the subject during the group decision making. Because decision makers have different knowledge backgrounds, practical experience, cognitive levels and preferences, the weight determination of the decision maker should reflect the importance and decision-making level of the decision maker objectively and completely. In the process of group decision-making, both the convergence of basic interests and objectives and conflicts of interests and preferences exist between decision makers. To help decision makers reach consensuses on decisionmaking problems, judgments should be made based on the full consideration of subjective factors and objective facts. This paper adopts the method of case-based learning to calculate the attribute weight in each decision makers' circumstance, thus to meet subjective preferences of different decision makers; optimizes the weight of each decision maker with the fuzzy set theory, and determines the level boundary between multiple decision makers to meet objective requirements during group decision-making; finally figures out the total order relation of the alternative which not only conforms to the subjective preference information of the decision maker, but also consists with the objective requirement of group decision-making, and seeks for consistent or compromised satisfactory alternatives among multiple subjects of which the boundary was unclear originally, to make group decision-making more objective and reasonable, and effectively integrates subjective judgment preferences of decision makers, which have a certain theoretical value and practical significance.

\section{Acknowledgments}

The authors would like to thank the guest editor and five anonymous referees for their valuable comments and suggestions. Their comments helped improve the quality of the paper immensely. The work was supported by the National Natural Science Foundation of China (71171112), KeyPrograms of Philosophy and Social Science Research in Colleges and Universities in Jiangsu Province,China (2012ZDIXM007), Key Programs of Key Research Bases of Philosophy and Social Science Research in Colleges and Universities in Jiangsu Province, China (2012JDXM003), Philosophy and Social Science Fund in Colleges and Universities in Jiangsu Province, China (2010SJD880040), Scientific and Technological Innovation Fund for Youth of Nanjing University of Aeronautics and Astronautics, China (Humanities and Social Sciences Category) (NR2012033), Fundamental Research Funds for the Central Universities of China (NS2014086).

\section{References}

[1] Ji. Yan and J. Liang, "A method of consensus analysis in hybrid multiple attribute group decision making", Chinese journal of management science, vol. 19, no. 6, (2011), pp. 133-140.

[2] Z. S. Xu, "A method based on distance measure for interval-valued intuitionistic fuzzy group decision making", Information Sciences, vol. 180, no. 1, (2010), pp. 181-190.

[3] D. Hector, C. Christensen and P. Jim, "A problem-structuring method for complex societal decisions:Its philosophical and psychological dimensions", European Journal of Operational Research, vol. 193, (2009), pp. 693-708.

[4] R. K. Kaushal and A. K Nema, "Multi-stakeholder decision analysis and comparative risk assessment for reuse-recycle oriented e-waste management strategies: A game theoretic approach, Waste Management and Research, vol. 31, no. 9, (2013), pp. 881-895.

[5] B. Wang and Q. Li, "Analysis of multi stakeholder game operations and management of toll road in Franchising", Statistics and decision, vol. 18, no. 16, (2013), pp. 44-51.

[6] C. Markmann, I. L. Darkow and C. D. Gracht, "A Delphi-based risk analysis-Identifying and assessing future challenges for supply chain security in a multi-stakeholder environment,Technological Forecasting and Social Change, vol. 80, no. 9, (2013), pp.1815-1833. 
[7] S. R. Agha, M. H. Jarbo and S. J. Matr, "A multi-criteria multi-stakeholder industrial projects prioritization in Gaza Stip",Arabian Journal for Science and Engineering, vol. 38, no. 5, (2013), pp. 1217-1227.

[8] Y. Guo and P. Yi, "Multi-attribute Decision Making in View of Degree fo Preference for Part of Alternatives", Journal of Northeastern University (Natural Science), vol. 28, no. 12, (2007), pp. 17821785.

[9] H. Wang, J. Zhu and Z. Fang, "Grey target cluster decision method on linguistic evaluation case-based, Journal of Mathematical Economics”, vol. 33, no. 12, (2013), pp. 3172-3181.

[10] J. Li, C. Yue and W. Li, "A dominance relation-based decision making approach for multi-attribute decision making problems with incomplete information", Control and Decision, vol. 28, no. 2, (2013), pp. 229-234.

[11] Y. J. Wang, "A fuzzy multi-criteria decision-making model by associating technique for order preference by similarity to ideal solution with relative preference relation", Information Sciences, vol. 268, no. 1, (2014), pp. 169-184.

[12] X. Zhang and C. Zhu, "Generalized precedence order method with ranking preference for multi-attribute decision making", Systems Engineering-Theory \& Practice, vol. 11, no. 1, (2013), pp. 2852-2858.

[13] F. Cai, X. Liao and N. Yang, "Multi-criteria sorting method based on assignment examples in the group decision context", Journal of management sciences in China, vol. 16, no. 2, (2013), pp. 22-32.

[14] A. Giarlotta and S. Greco, "Necessary and possible preference structures", Journal of Mathematical Economics, vol. 49, (2013), pp. 163-172.

[15] S. Y. Huang, X. Y. Su and Y. Hu, S. Mahadevan and Y. Deng, "A new decision-making method by incomplete preferences based on evidence distance", Knowledge-Based Systems, vol. 56, no. 2, (2014), pp. 264-272.

[16] X. Sun and X. Feng, "Expert classification and aggregation method for group decision-making based on judgment similarity degree", Operations research and management science, vol. 23, no. 1, (2014), pp. $52-58$.

[17] J. Xu and F. Shen, "A new outranking choice method for group decision making under Atanassov's interval-valued intuitionistic fuzzy environment”, Knowledge-Based Systems, vol. 70, no. 11, (2014), pp. 177-188.

[18] R. Zhou, F. Fan, D. He and W. Qiu, "Integrated entropy weight method based on data stability and subjective preference in multi-attribute group decision-making", Control and Decision, vol. 27, no. 8, (2012), pp. 1169-1174.

[19] P. Eklund, A. Rusinowska and H. D. Swart, "Consensus reaching in-committees, European Journal of Operational Research", vol. 178, (2007), pp. 185-193.

[20] L. He, L.Wang and L. Zhang, "The weight optimal choice method based on experts' weights clustering analyses in multi-resource leveling optimization", System engineering, vol. 32, no. 12, (2014), pp. 124132.

[21] Z. X. Xiaolu, "Deriving experts' weights based on consistency maximization in intuitionistic fuzzy group decision making”, Journal of Intelligent and Fuzzy Systems, vol. 27, no. 1, (2014), pp. 221-233.

[22] X. Sun and S. Zeng, "A Method Based on Authority and Consistency for Determining Experts' Weights in Group Comprehensive Evaluation", Mathmatics in practice and theory, vol. 44, no. 8, (201), pp. 4247.

[23] Zhongliang, "Approach to group decision making based on determining the weights of experts by using projection method", Applied Mathematical Modelling, vol. 36, no. 7, (2012), pp. 2900-2910.

[24] J. Song, Y. Dang, Z. Wang and K. Zhang, "New decision model of grey target with both the positive clout and the negative clout", Journal of Mathematical Economics, vol. 30, no. 10, (2010), pp. 18221827.

[25] L. Yu, S. Wang and K. K. Lai, "An intelligent-agent-based fuzzy group decision making model for financial multicriteria decision support:The case of credit scoring", European journal of operational research, vol. 195, (2009), pp. 942-959.

\section{Authors}

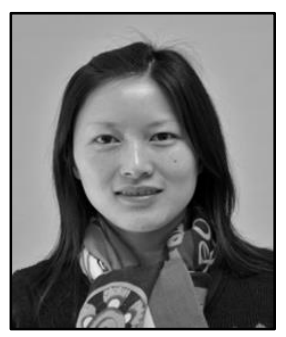

Dai Quanchen, received her master degree in business administration from Nanjing University of Aeronautics and Astronautics in Nanjing, China. She is currently a research assistant in the College of Economics and Management in NUAA. Her research interest is mainly in the area of decision theory and method, group decision, grey system theory. She has published several research papers in scholarly journals in the above research areas. 
International Journal of $u-$ and e- Service, Science and Technology Vol.9, No. 8 (2016) 